\title{
Entropy versus Volume for Pseudo-Anosovs
}

\author{
E. Kin, S. Kojima, and M. Takasawa
}

\section{CONTENTS}

1. Introduction

2. Pseudo-Anosovs

3. Linear Bounds for Entropy versus Volume

4. Generating Sets of Mapping Class Groups

5. Minimal Dilatation and Minimal Volume

6. Lower Bounds for ent/vol

Acknowledgments

References
2000 AMS Subject Classification: Primary 37E30, 57M27; Secondary 57M55

Keywords: Mapping class group, braid group, pseudo-Anosov, dilatation, entropy, hyperbolic volume
We discuss a comparison of the entropy of pseudo-Anosov maps and the volume of their mapping tori. Recent study of the WeilPetersson geometry of Teichmüller space tells us that the entropy and volume admit linear inequalities for both directions under some bounded geometry condition. Based on experiments, we present various observations on the relation between minimal entropies and volumes, and on bounding constants for the entropy over the volume from below. We also provide explicit bounding constants for a punctured torus case.

\section{INTRODUCTION}

Let $\Sigma=\Sigma_{g, p}$ be an orientable surface of genus $g$ with $p$ punctures and $\mathcal{M}(\Sigma)$ the mapping class group of $\Sigma$. Assume that $3 g-3+p \geq 1$. According to [Thurston 88], the elements of $\mathcal{M}(\Sigma)$ are classified into three types: periodic, pseudo-Anosov, and reducible. A pseudo-Anosov element $\phi$ of $\mathcal{M}(\Sigma)$ defines two natural numeric invariants. One is the entropy ent $(\phi)$, which is the logarithm of the stretching factor of the invariant foliation of $\phi$ (often called the dilatation of $\phi$ ). The other is the volume $\operatorname{vol}(\phi)$ of its mapping torus,

$$
\mathbb{T}(\phi)=\Sigma \times[0,1] / \sim
$$

with respect to the hyperbolic metric, whose the existence is due to [Thurston 98], and the uniqueness, to Mostow rigidity. Here, $\sim$ identifies $(x, 1)$ with $(f(x), 0)$ for some representative $f$ of $\phi$.

Our study is motivated by experiments of the lastnamed author, illustrated in Figure 1, in his thesis [Takasawa 00] comparing $\operatorname{ent}(\phi)$ and $\operatorname{vol}(\phi)$. To see this more precisely, we let $\mathcal{M}^{\mathrm{pA}}(\Sigma)$ be the set of pseudo-Anosov mapping classes of $\mathcal{M}(\Sigma)$ and put

$$
\mathcal{E}(\Sigma)=\left\{(\operatorname{vol}(\phi), \operatorname{ent}(\phi)) \in \mathbb{R}^{2} \mid \phi \in \mathcal{M}^{\mathrm{pA}}(\Sigma)\right\} .
$$

Figure 1 is the plot of $\mathcal{E}\left(\Sigma_{2,0}\right)$ for all pseudo-Anosov classes represented by words of length at most 7 with respect to the Lickorish generators. From this plot, one might suspect that the ratios $\operatorname{ent}(\phi) / \operatorname{vol}(\phi)$ are bounded 


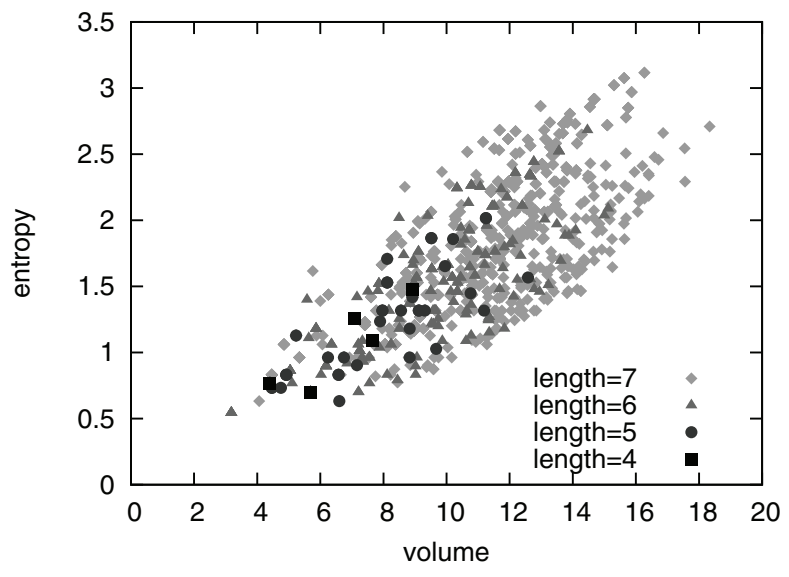

FIGURE 1. Entropy versus volume for $\Sigma_{2,0}$.

for both directions, namely, that there is a constant $C$ depending only on the topology of $\Sigma$ satisfying

$$
C^{-1} \operatorname{vol}(\phi) \leq \operatorname{ent}(\phi) \leq C \operatorname{vol}(\phi)
$$

However, this is false in general, since it has been known to the experts from [Long and Morton 86] and [Fathi 87] that there are many families of pseudo-Anosov maps whose entropies tend to infinity while volumes remain bounded. We will present more recent plots in which we can observe this fact in Section 4.

Nevertheless, it is still reasonable to expect under some bounded geometry condition that the ratios $\operatorname{ent}(\phi) / \operatorname{vol}(\phi)$ are bounded for both directions, because the families in [Long and Morton 86, Fathi 87] necessarily contain short geodesics asymptotically. As we will explain in Section 3, our expectation turns out to be a consequence of the deep results to be found in [Minsky 93], [Brock 03], and the recent work [Brock et al. 09] of Brock, Mazur, and Minsky.

On the other hand, the theory above does not say very much about accurate values of bounding constants. Experiments should provide a more practical working hypothesis. From the computational viewpoint, it is rather easy to work not with closed surfaces but with punctured disks, since they have nice descriptions in terms of braid data. Let $D_{n}$ be an $n$-punctured disk. See Figure 2 for a more accurate plot for the case of $D_{6}$.

The purpose of this paper is to present various observations and problems based on our experiments for the relation between minimal entropies and volumes, and for constants bounding the ratio ent/vol from below for pseudo-Anosov maps on punctured disks and tori. More-

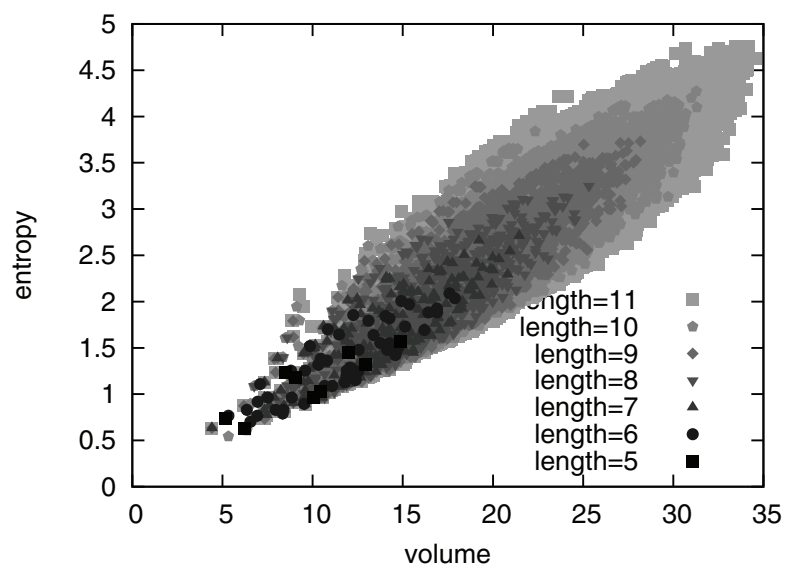

FIGURE 2. Entropy versus volume for $D_{6}$.

over, we prove in Theorem 6.7 that

$$
\frac{\operatorname{ent}(\phi)}{\operatorname{vol}(\phi)}>\frac{\log \left(\frac{3+\sqrt{5}}{2}\right)}{2 v_{8}} \approx 0.1313
$$

for any $\phi \in \mathcal{M}^{\mathrm{pA}}\left(\Sigma_{1,1}\right)$, where $v_{8} \approx 3.6638$ is the volume of a regular ideal octahedron. This bound is not best possible, unfortunately. However, restricting our attention to mapping classes of block length 1 , we obtain in Proposition 6.8 the best possible lower bound

$$
\frac{\log \left(\frac{3+\sqrt{5}}{2}\right)}{2 v_{3}} \approx 0.4741
$$

where $v_{3} \approx 1.0149$ is the volume of a regular ideal tetrahedron.

The organization of this paper is as follows. After recalling a basis of pseudo-Anosovs in the next section, we explain in Section 3 how the linear inequalities (1-1) are derived from recent studies of the Teichmüller space. Then choosing preferred generating sets of $\mathcal{M}(\Sigma)$ in Section 4 for the experiments, we discuss the relation between minimal entropies and volumes in Section 5, and lower bounds for ent/vol together with more accurate bounds of a special case in Section 6 .

\section{PSEUDO-ANOSOVS}

The mapping class group $\mathcal{M}(\Sigma)$ is the group of isotopy classes of orientation-preserving homeomorphisms of $\Sigma$, where the group operation is induced by composition of homeomorphisms. An element of the mapping class group is called a mapping class.

A homeomorphism $\Phi: \Sigma \rightarrow \Sigma$ is said to be pseudoAnosov if there exist a constant $\lambda=\lambda(\Phi)>1$, called 
the dilatation of $\Phi$, and a pair of transverse measured foliations $\mathcal{F}^{s}$ and $\mathcal{F}^{u}$ such that

$$
\Phi\left(\mathcal{F}^{s}\right)=\frac{1}{\lambda} \mathcal{F}^{s} \quad \text { and } \quad \Phi\left(\mathcal{F}^{u}\right)=\lambda \mathcal{F}^{u}
$$

A mapping class that contains a pseudo-Anosov homeomorphism is called pseudo-Anosov. We define the dilatation of a pseudo-Anosov mapping class $\phi$, denoted by $\lambda(\phi)$, to be the dilatation of a pseudo-Anosov representative in $\phi$. It can be verified that $\lambda(\phi)$ does not depend on the choice of representative.

Fixing $\Sigma$, the dilatation $\lambda(\phi)$ for $\phi \in \mathcal{M}^{\mathrm{pA}}(\Sigma)$ is known to be an algebraic integer with a bounded degree depending only on $\Sigma$. Also, the number of conjugacy classes of $\mathcal{M}^{\mathrm{pA}}(\Sigma)$ with dilatations bounded by some constant is finite. In particular, there exists a pseudoAnosov with least dilatation $\lambda(\Sigma)$; see [Ivanov 90].

The topological entropy ent $(f)$ of a continuous self-map $f$ on a compact metric space is a measure of the complexity; see, for instance, [Walters 82]. For a pseudo-Anosov homeomorphism $\Phi$, the equality $\operatorname{ent}(\Phi)=\log (\lambda(\Phi))$ holds [Fathi et al. 79] and $\operatorname{ent}(\Phi)$ attains the minimal entropy among all homeomorphisms that are isotopic to $\Phi$. Thus, we denote by $\operatorname{ent}(\phi)$ this characteristic number associated to a pseudo-Anosov mapping class $\phi$.

Choosing a representative $f: \Sigma \rightarrow \Sigma$ of $\phi \in \mathcal{M}(\Sigma)$, we form a mapping torus

$$
\mathbb{T}(\phi)=\Sigma \times[0,1] / \sim
$$

where $\sim$ identifies $(x, 1)$ with $(f(x), 0)$. Then $\phi$ is pseudoAnosov if and only if $\mathbb{T}(\phi)$ admits a complete hyperbolic structure of finite volume [Thurston 98, Otal and Kay 01]. Since such a structure is unique up to isometry by Mostow rigidity, it makes sense to speak of the volume $\operatorname{vol}(\phi)$ of $\phi$, the hyperbolic volume of $\mathbb{T}(\phi)$.

Remark 2.1. Let $\phi$ be a pseudo-Anosov homeomorphism on $\Sigma$. Then the identities

$$
\operatorname{vol}\left(\phi^{m}\right)=m \operatorname{vol}(\phi) \quad \text { and } \quad \operatorname{ent}\left(\phi^{m}\right)=m \operatorname{ent}(\phi)
$$

hold for any positive integer $m$. In particular, the line with slope ent $(\phi) / \operatorname{vol}(\phi)$ in $\mathbb{R}^{2}$ passing through the origin must intersect $\mathcal{E}(\Sigma)$ in infinitely many points.

\section{LINEAR BOUNDS FOR ENTROPY VERSUS VOLUME}

We here briefly describe what can be known about entropy versus volume for $\mathcal{M}^{\mathrm{pA}}(\Sigma)$ from the very recent theory. To see this, we introduce two norms for a pseudoAnosov $\phi$. Let $\|\phi\|_{*}$ be the minimal translation distance of the action of $\phi$ on the Teichmüller space with respect to the Teichmüller distance $d_{\mathrm{T}}$ or the Weil-Petersson distance $d_{\mathrm{WP}}$ according to whether $*=\mathrm{T}$ or WP. Notice that

$$
\operatorname{ent}(\phi)=\|\phi\|_{\mathrm{T}}
$$

We start with a result in [Brock 03] showing that there is a universal constant $D$ depending only on the topology of $\Sigma$ such that the inequalities

$$
D^{-1} \operatorname{vol}(\phi) \leq\|\phi\|_{\mathrm{WP}} \leq D \operatorname{vol}(\phi)
$$

hold for any pseudo-Anosov $\phi$ on $\Sigma$. To get (1-1), we want to replace $\|\cdot\|_{\mathrm{WP}}$ by $\|\cdot\|_{\mathrm{T}}$ under some bounded condition.

The Teichmüller distance is originally defined using the infimum of dilatations of quasiconformal maps between two Riemann surfaces. On the other hand, the Weil-Petersson distance is defined as the associated distance with the Riemannian part $g_{\mathrm{WP}}$ of the WeilPetersson metric. Linch succeeded in obtaining a comparison of two distances directly; see [Linch 74]. The modern treatment of the Teichmüller distance, which can be found, for instance, in [Gardiner and Lakic 00], introduces an infinitesimal interpretation $g_{\mathrm{T}}$ of $d_{\mathrm{T}}$. Then the infinitesimal form of Linch's inequality,

$$
g_{\mathrm{WP}} \leq-2 \pi \chi(\Sigma) g_{\mathrm{T}}
$$

can be derived simply from the Cauchy-Schwarz inequality between norms whose duals define infinitesimal forms of two distances; see, for instance, [Royden 74].

Now choose a point $x$ on the Teichmüller geodesic of $\phi$. Then since $x$ may not be on the Weil-Petersson geodesic of $\phi$, we have

$$
\begin{aligned}
\|\phi\|_{\mathrm{WP}} & \leq d_{\mathrm{WP}}(x, \phi(x)) \leq-2 \pi \chi(\Sigma) d_{\mathrm{T}}(x, \phi(x)) \\
& =-2 \pi \chi(\Sigma)\|\phi\|_{\mathrm{T}} .
\end{aligned}
$$

This together with the left inequality of (3-1) immediately implies the left inequality in $(1-1)$.

The right inequality in (1-1) does not hold in general, as we mentioned in the introduction. However, the deep analysis carried out for the Teichmüller distance in [Minsky 93] and for the Weil-Petersson distance in [Brock et al. 09] implies, among many others things, the following.

Theorem 3.1. [Minsky 93, Brock et al. 09] For any $\varepsilon>0$, there exists $\delta>0$ such that both the Teichmüller and the Weil-Petersson geodesics invariant under the action of 

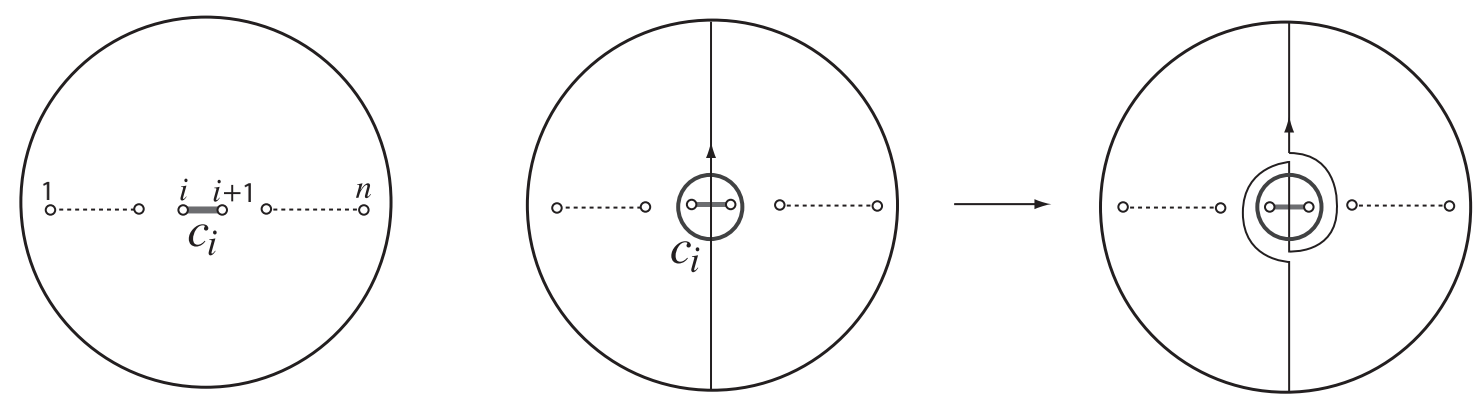

FIGURE 3. Arc $c_{i}$ and positive half-twist $h_{c_{i}}$.

a pseudo-Anosov $\phi$ have no intersection with the subset of the Teichmüller space consisting of hyperbolic surfaces with closed geodesic of length $<\delta$ if $\mathbb{T}(\phi)$ contains no closed geodesics of length less than $\varepsilon$.

Now, since the part of the Teichmüller space which consists of thick surfaces appearing above is invariant under the action of the mapping class group, and moreover, the quotient is compact by Mumford, we obtain the oppositely directed inequality to (3-2) within this region. Namely, there is some constant $A$ such that the inequality

$$
g_{\mathrm{T}} \leq A g_{\mathrm{WP}}
$$

holds in the region of the Teichmüller space consisting of surfaces without closed geodesics of length less than $\delta$.

Choose $y$ on the Weil-Petersson geodesic of $\phi$. Then we have

$$
\|\phi\|_{\mathrm{T}} \leq d_{\mathrm{T}}(x, \phi(x)) \leq A d_{\mathrm{WP}}(x, \phi(x))=A\|\phi\|_{\mathrm{WP}} .
$$

This together with the right inequality in (3-1) implies that of (1-1). Thus we have the following corollary to [Brock 03, Minsky 93, Brock et al. 09].

Theorem 3.2. There exists a constant $B=B(\Sigma)$ depending only on the topology of $\Sigma$ such that the inequality

$$
B \operatorname{vol}(\phi) \leq \operatorname{ent}(\phi)
$$

holds for any pseudo-Anosov $\phi$ on $\Sigma$. Furthermore, for any $\varepsilon>0$, there exists a constant $C=C(\varepsilon, \Sigma)>1$ depending only on $\varepsilon$ and the topology of $\Sigma$ such that the inequality

$$
\operatorname{ent}(\phi) \leq C \operatorname{vol}(\phi)
$$

holds for any pseudo-Anosov $\phi$ on $\Sigma$ whose mapping torus $\mathbb{T}(\phi)$ has no closed geodesics of length less than $\varepsilon$.

Remark 3.3. The later half of Theorem 3.2 says that a sequence of pseudo-Anosov maps whose entropies diverge while volumes remain bounded must contain short geodesics asymptotically. The sequences found in [Long and Morton 86, Fathi 87] in fact have this property. The number of samples in the experiments plotted in Figure 1 was insufficient to exhibit such a sequence.

\section{GENERATING SETS OF MAPPING CLASS GROUPS}

In this section we shall exhibit a preferred generating set that we use for the experimental plots of entropy versus volume for $\Sigma=D_{n}$ and $\Sigma_{n, p}$, where $(n, p)=(1,1)$ or $(2,0)$.

First of all, we introduce a generating set of the mapping class group $\mathcal{M}\left(D_{n}\right)$ by

$$
\left\{h_{c_{1}}, \ldots, h_{c_{n-1}}\right\}
$$

where $h_{c_{i}}$ denotes the mapping class that represents the positive half-twist about the $\operatorname{arc} c_{i}$ from the $i$ th puncture to the $(i+1)$ st; see Figure 3 .

The $n$-braid group $B_{n}$ and the mapping class group $\mathcal{M}\left(D_{n}\right)$ are related by the surjective homomorphism

$$
\begin{array}{rll}
\Gamma: B_{n} & \rightarrow & \mathcal{M}\left(D_{n}\right) \\
\Psi & & \Psi \\
\sigma_{i} & \mapsto & h_{c_{i}}
\end{array}
$$

where $\sigma_{i}$ for $i \in\{1, \ldots, n-1\}$ is the Artin generator; see Figure 4.

The kernel of $\Gamma$ is the center of $B_{n}$, which is generated by the full-twist braid $\left(\sigma_{1} \sigma_{2} \cdots \sigma_{n-1}\right)^{n}$. Note that $\mathcal{M}\left(D_{n}\right)$ is isomorphic to a subgroup of $\mathcal{M}\left(\Sigma_{0, n+1}\right)$ through identification of the boundary of $D_{n}$ with the $(n+1)$ st puncture.

In the rest of the paper, we regard a mapping class in $\mathcal{M}\left(D_{n}\right)$ as a mapping class in $\mathcal{M}\left(\Sigma_{0, n+1}\right)$ by fixing the $(n+1)$ st puncture.

We say that a braid $b \in B_{n}$ is pseudo-Anosov if $\Gamma(b) \in \mathcal{M}\left(D_{n}\right)$ is pseudo-Anosov. When this is the case, 


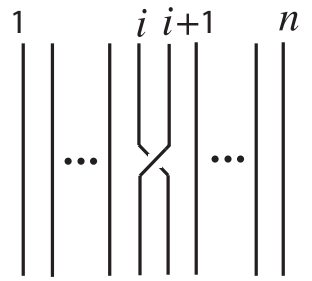

FIGURE 4. Generator $\sigma_{i}$.

$\operatorname{vol}(\Gamma(b))$ equals the hyperbolic volume of the link complement $S^{3} \backslash \bar{b}$ in the 3 -sphere $S^{3}$, where $\bar{b}$ is the braided link of $b$ that is the union of the closed braid of $b$ and the braid axis; see Figure 5 .

Hereinafter we represent a mapping class in $\mathcal{M}\left(D_{n}\right)$ by a braid, and we denote $\Gamma(b) \in \mathcal{M}\left(D_{n}\right)$ by $b$.

As for $\Sigma_{n, p}$, where $(n, p)=(1,1)$ or $(2,0)$, we choose the set of Lickorish generators as a preferred generating set. Let us describe them more precisely. Let $\tau_{1}$ and $\tau_{2}$ be positive Dehn twists along respectively a meridian and a longitude for $\Sigma_{1,1}$. Then the set

$$
\left\{\tau_{1}, \tau_{2}\right\}
$$

will be a preferred generating set for $\mathcal{M}\left(\Sigma_{1,1}\right)$. The elements $\tau_{1}, \tau_{2}$ are related to $\sigma_{1}, \sigma_{2}$ in $\mathcal{M}\left(D_{3}\right)$ through a double cover of $D_{3}$ branched along three punctures and one hole, where the hole corresponds to the puncture in $\Sigma_{1,1}$.

Let us choose a set of five essential simple closed curves $\alpha_{1}, \alpha_{2}, \ldots, \alpha_{5}$ on $\Sigma_{2,0}$ such that $\alpha_{i} \cap \alpha_{j}$ is one point if $|i-j|=1$ and is empty otherwise, and $\tau_{i}$ is a positive Dehn twist along $\alpha_{i}$ for $i=1,2, \ldots, 5$. Then the set

$$
\left\{\tau_{1}, \tau_{2}, \ldots, \tau_{5}\right\}
$$

will be our preferred generating set for $\mathcal{M}\left(\Sigma_{2,0}\right)$.

Again the elements $\tau_{1}, \tau_{2}, \ldots, \tau_{4}$ are related to $\sigma_{1}, \sigma_{2}, \ldots, \sigma_{4}$ in $\mathcal{M}\left(D_{5}\right)$ through a double cover of $D_{5}$ branched along five punctures and one hole, where the hole corresponds to some point on $\Sigma_{2,0}$.

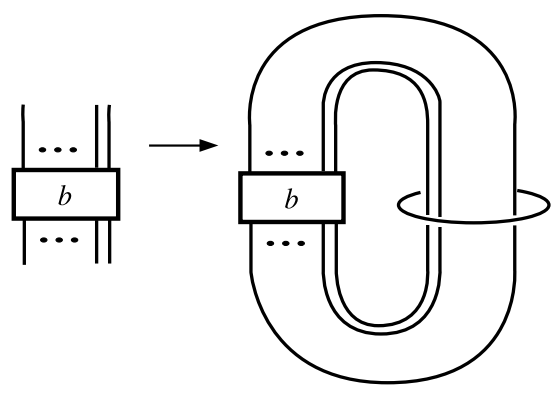

FIGURE 5. Link $\bar{b}$ obtained from a braid $b$.

\section{MINIMAL DILATATION AND MINIMAL VOLUME}

\subsection{Experimental Data}

For the exposition of our experimental data, we introduce the notation $\mathcal{E}_{k}(\Sigma)$ for the subset of $\mathcal{E}(\Sigma)$ formed by pseudo-Anosovs of word length at most $k$ with respect to the preferred generating set. Namely,

$$
\begin{gathered}
\mathcal{E}_{k}(\Sigma)=\left\{(\operatorname{vol}(\phi), \operatorname{ent}(\phi)) \mid \phi \in \mathcal{M}^{\mathrm{pA}}(\Sigma)\right. \\
\text { of word length } \leq k\} .
\end{gathered}
$$

Figures 6-9 are respectively the plots of $\mathcal{E}_{15}\left(D_{3}\right)$, $\mathcal{E}_{12}\left(D_{4}\right), \mathcal{E}_{10}\left(D_{5}\right)$, and $\mathcal{E}_{11}\left(D_{6}\right)$. We use the program by $\mathrm{T}$. Hall ${ }^{1}$ for the computation of braid dilatations, and SnapPea by J. Weeks ${ }^{2}$ for the computation of volumes of links in the 3 -sphere $S^{3}$.

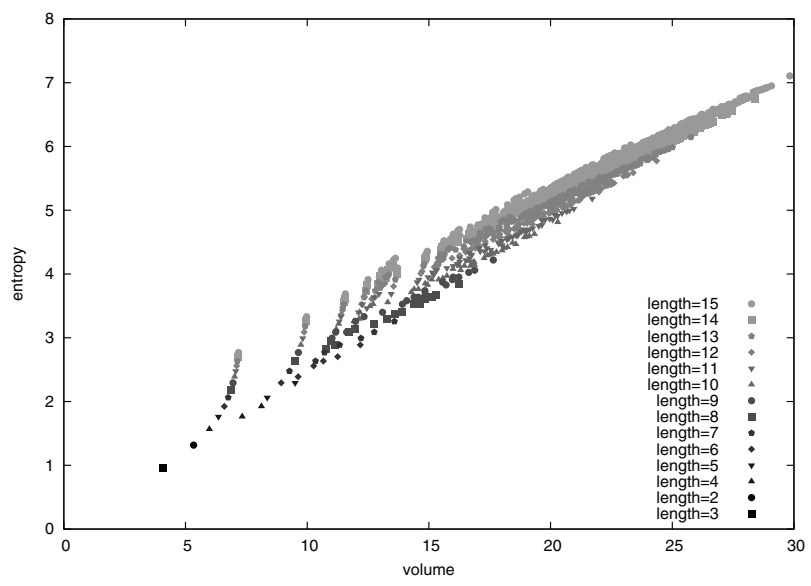

FIGURE 6. $\mathcal{E}_{15}\left(D_{3}\right)$.

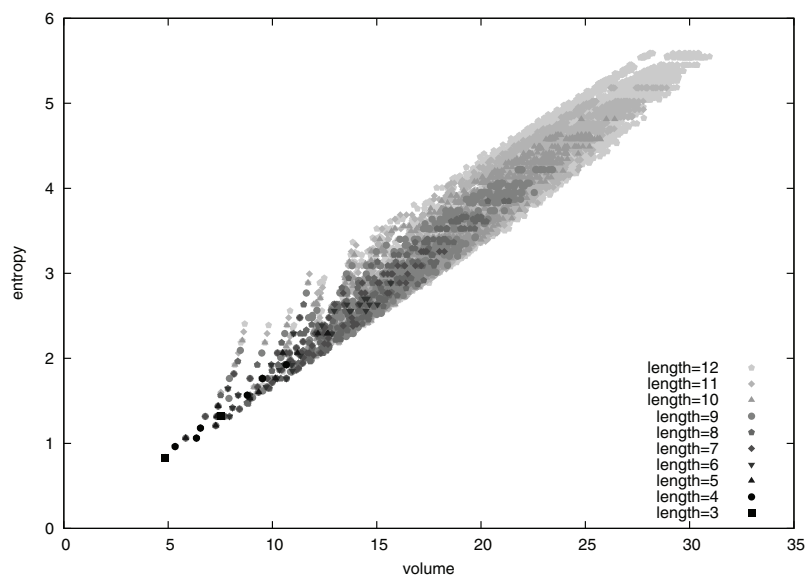

FIGURE 7. $\mathcal{E}_{12}\left(D_{4}\right)$.

\footnotetext{
${ }^{1}$ Available online (http://www.liv.ac.uk/maths/PURE/MIN_ SET/CONTENT/members/T_Hall.html).

${ }^{2}$ Available online (http://www.geometrygames.org/SnapPea/).
} 


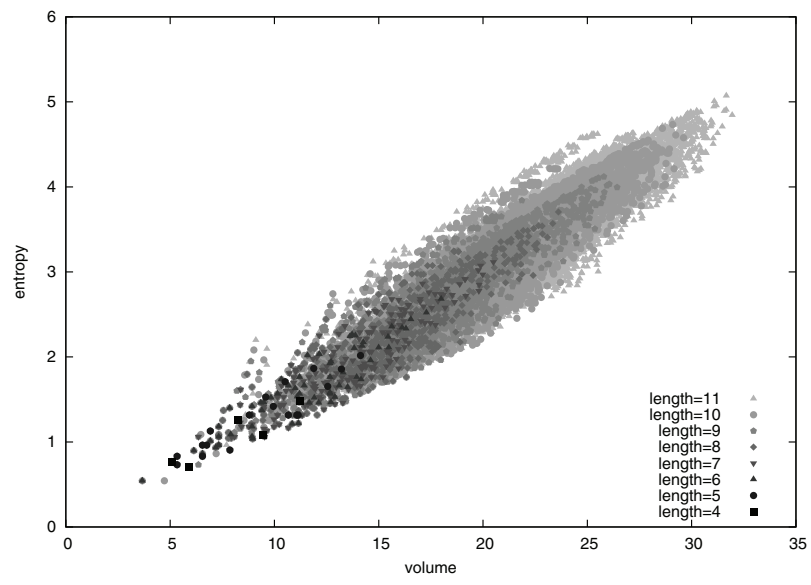

FIGURE 8. $\mathcal{E}_{10}\left(D_{5}\right)$

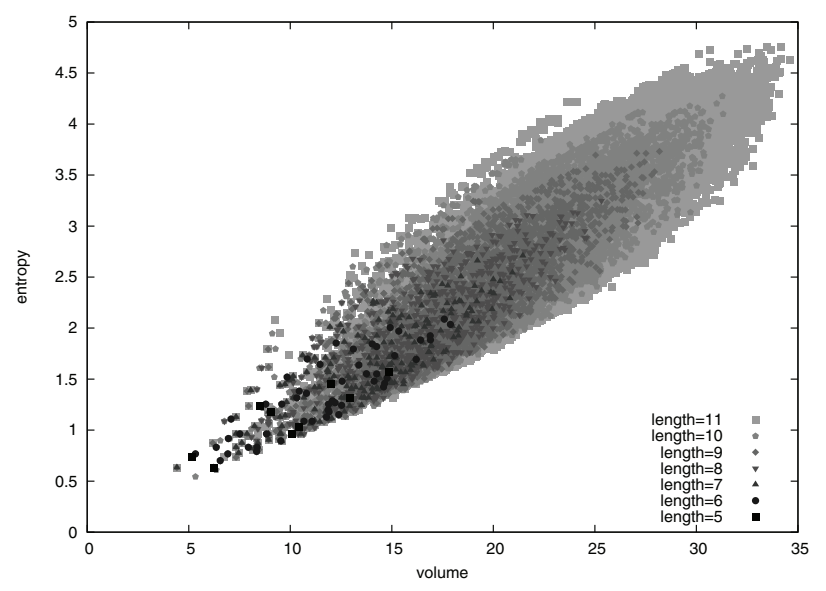

FIGURE 9. $\mathcal{E}_{11}\left(D_{6}\right)$.

\subsection{Observations}

Recall that $\lambda(\Sigma)$ represents the minimal dilatation among $\lambda(\phi)$ for $\phi \in \mathcal{M}^{\mathrm{pA}}(\Sigma)$. In addition to $\lambda(\Sigma)$, we introduce the following further notation for the exposition:

$$
\begin{gathered}
\lambda_{k}(\Sigma)=\min \left\{\lambda(\phi) \mid \phi \in \mathcal{M}^{\mathrm{pA}}(\Sigma) \text { of word length } \leq k\right\}, \\
\lambda(\Sigma ; c)=\min \left\{\lambda(\phi) \mid \phi \in \mathcal{M}^{\mathrm{pA}}(\Sigma), \mathbb{T}(\phi) \text { has } c \text { cusps }\right\}, \\
\lambda_{k}(\Sigma ; c)=\min \left\{\lambda(\phi) \mid \phi \in \mathcal{M}^{\mathrm{pA}}(\Sigma)\right. \text { of word length } \\
\leq k, \mathbb{T}(\phi) \text { has } c \text { cusps }\} .
\end{gathered}
$$

When $\Sigma=D_{n}$, the number of cusps of $\mathbb{T}(b)$ for $b \in$ $\mathcal{M}^{\mathrm{pA}}\left(D_{n}\right)$ equals the number of the components of the link $\bar{b}$, since $\mathbb{T}(b)=S^{3} \backslash \bar{b}$.

The minimal dilatation $\lambda(\Sigma)$ and the minimal entropy $\operatorname{ent}(\Sigma)=\log \lambda(\Sigma)$ are known for the surfaces in Table 1.

We now turn to the volume. The set of volumes of hyperbolic 3-manifolds, called the volume spectrum, is known to be a well-ordered closed subset in $\mathbb{R}$ of order type $\omega^{\omega}$; see [Thurston 79]. In particular, any subset of the volume spectrum achieves its infimum. We set, again for the exposition,

$$
\begin{aligned}
& \operatorname{vol}(\Sigma)=\min \left\{\operatorname{vol}(\phi) \mid \phi \in \mathcal{M}^{\mathrm{pA}}(\Sigma)\right\} \\
& \operatorname{vol}_{k}(\Sigma) \\
& =\min \left\{\operatorname{vol}(\phi) \mid \phi \in \mathcal{M}^{\mathrm{pA}}(\Sigma) \text { of word length } \leq k\right\} \\
& \operatorname{vol}(\Sigma ; c) \\
& =\min \left\{\operatorname{vol}(\phi) \mid \phi \in \mathcal{M}^{\mathrm{pA}}(\Sigma), \mathbb{T}(\phi) \text { has } c \text { cusps }\right\} \\
& \operatorname{vol}_{k}(\Sigma ; c) \\
& =\min \left\{\operatorname{vol}(\phi) \mid \phi \in \mathcal{M}^{\mathrm{pA}}(\Sigma) \text { of word length } \leq k,\right. \\
& \qquad \mathbb{T}(\phi) \text { has } c \text { cusps }\}
\end{aligned}
$$

To discuss which mapping class reaches $\lambda(\Sigma)$ and which one reaches $\operatorname{vol}(\Sigma)$, we first confirm that there exists a mapping class simultaneously reaching both $\lambda(\Sigma)$ and $\operatorname{vol}(\Sigma)$ when $\Sigma=D_{3}$ or $D_{5}$. It is shown in [Guéritaud 06, Theorem B.1] that the 3-braid $\beta_{3}=\sigma_{1} \sigma_{2}^{-1}$ with the minimal dilatation appearing in Table 1 realizes $\operatorname{vol}\left(D_{3}\right) \approx 4.05976$. The braided link $\overline{\beta_{5}}$ of the 5 -braid $\beta_{5}=\sigma_{1}^{3} \sigma_{2} \sigma_{3} \sigma_{4}$ with minimal dilatation appearing in Table 1 equals the $(-2,3,8)$-pretzel link; see Figure 10 . On the other hand, it is shown in [Agol 08] that the $(-2,3,8)$-pretzel link complement and the Whitehead link complement have minimal volume among orientable 2 -cusped hyperbolic 3 -manifolds. Hence $\beta_{5}$ also realizes $\operatorname{vol}\left(D_{5}\right) \approx 3.66386$.

Thus one may ask whether there exists a mapping class simultaneously reaching both $\lambda(\Sigma)$ and $\operatorname{vol}(\Sigma)$. However, this seems to be false in general. Within our experiments, $\operatorname{vol}_{11}\left(D_{6}\right)$ and $\lambda_{11}\left(D_{6}\right)$ are not reached by the same mapping class. This may be caused by the fact that the mapping torus reaching $\operatorname{vol}_{11}\left(D_{6}\right)$ and the one reaching $\lambda_{11}\left(D_{6}\right)$ have different numbers of cusps. Thus, we propose a refined problem by taking the number of cusps into account.
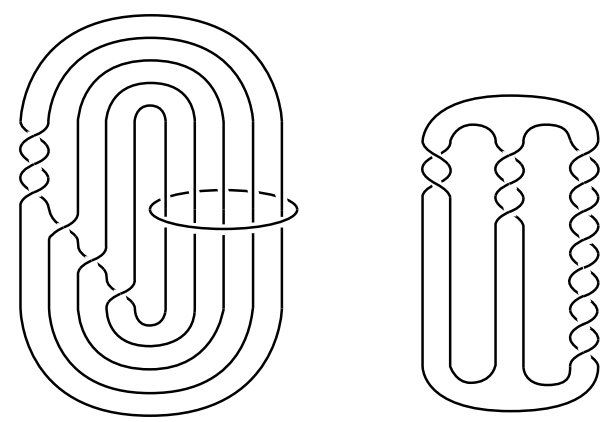

FIGURE 10. Link $\overline{\beta_{5}}$ on the left is equal to the $(-2,3,8)$-pretzel link on the right. 


\begin{tabular}{|l|c|c|c|c|}
\hline $\boldsymbol{\Sigma}$ & $\boldsymbol{\lambda}(\boldsymbol{\Sigma})$ & $\operatorname{ent}(\boldsymbol{\Sigma})$ & Mapping Class Realizing $\boldsymbol{\lambda}(\boldsymbol{\Sigma})$ & Reference \\
\hline$\Sigma_{1,1}$ & $\approx 2.61803$ & $\approx 0.96242$ & $\tau_{1} \tau_{2}^{-1}$ & folklore \\
$D_{3}$ & $\approx 2.61803$ & $\approx 0.96242$ & $\beta_{3}:=\sigma_{1} \sigma_{2}^{-1}$ & [Matsuoka 86] \\
$D_{4}$ & $\approx 2.29663$ & $\approx 0.83144$ & $\beta_{4}:=\sigma_{1} \sigma_{2} \sigma_{3}^{-1}$ & [Ko et al. 02] \\
$D_{5}$ & $\approx 1.72208$ & $\approx 0.54353$ & $\beta_{5}:=\sigma_{1}^{3} \sigma_{2} \sigma_{3} \sigma_{4}$ & [Ham and Song 07] \\
$\Sigma_{2,0}$ & $\approx 1.72208$ & $\approx 0.54353$ & $\tau_{1}^{3} \tau_{2} \tau_{3} \tau_{4}$ & [Cho and Ham 08] \\
\hline
\end{tabular}

TABLE 1. Minimal dilatations.

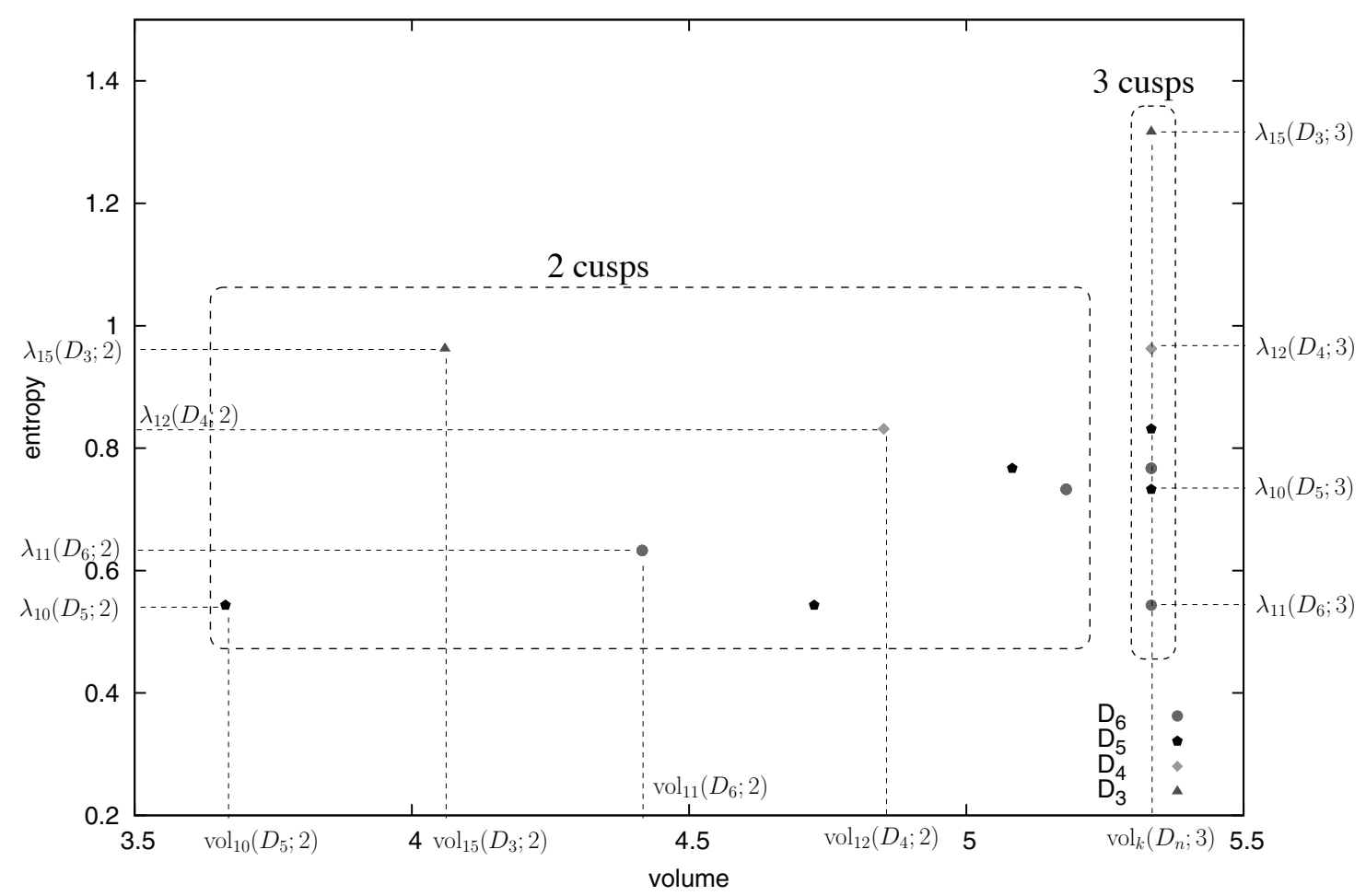

FIGURE 11. Samples with small entropy and small volume.

Problem 5.1. Does there exist a mapping class in $\mathcal{M}^{\mathrm{pA}}(\Sigma)$ reaching both $\lambda(\Sigma ; c)$ and $\operatorname{vol}(\Sigma ; c)$ simultaneously?

To view our experimental data more carefully for approaching this problem, we exhibit in Figure 11 the plots of

$$
\mathcal{E}_{15}\left(D_{3}\right), \quad \mathcal{E}_{12}\left(D_{4}\right), \quad \mathcal{E}_{10}\left(D_{5}\right), \quad \mathcal{E}_{11}\left(D_{6}\right)
$$

restricted to the range of the volume less than 5.334. We can observe the following supporting evidence for Problem 5.1:

1a. The 3-braid $\beta_{3}=\sigma_{1} \sigma_{2}^{-1}$ reaches both $\lambda\left(D_{3}\right) \approx$ 2.61803 and $\operatorname{vol}\left(D_{3}\right) \approx 4.05976$. Thus $\lambda\left(D_{3}\right)=$ $\lambda\left(D_{3} ; 2\right)$ and $\operatorname{vol}\left(D_{3}\right)=\operatorname{vol}\left(D_{3} ; 2\right)$. 1b. It is easy to verify that the 3 -braid $\sigma_{1}^{2} \sigma_{2}^{-1}$ reaches $\lambda\left(D_{3} ; 3\right)$. This 3 -braid also reaches $\operatorname{vol}_{15}\left(D_{3} ; 3\right)=$ $\operatorname{vol}\left(S^{3} \backslash C_{3}\right) \approx 5.33348$, where $C_{3}$ is the chain-link with three components; see Figure 12. Among orientable 3-cusped hyperbolic 3-manifolds, $S^{3} \backslash C_{3}$, which is called the magic manifold, is the one with the smallest known volume.

2a. The 4-braid $\beta_{4}=\sigma_{1} \sigma_{2} \sigma_{3}^{-1}$ reaches both $\lambda\left(D_{4}\right)=$ $\lambda\left(D_{4} ; 2\right) \approx 2.29663$ and $\operatorname{vol}_{12}\left(D_{4} ; 2\right) \approx 4.85117$.

2b. The 4-braid $\sigma_{1}^{2} \sigma_{2} \sigma_{3}^{-1}$ reaches both $\lambda_{12}\left(D_{4} ; 3\right) \approx$ 2.61803 and $\operatorname{vol}_{12}\left(D_{4} ; 3\right)=\operatorname{vol}\left(S^{3} \backslash C_{3}\right)$.

3a. The 5 -braid $\beta_{5}=\sigma_{1}^{3} \sigma_{2} \sigma_{3} \sigma_{4}$ reaches both $\lambda\left(D_{5}\right)=$ $\lambda\left(D_{5} ; 2\right) \approx 1.72208$ and $\operatorname{vol}\left(D_{5}\right)=\operatorname{vol}\left(D_{5} ; 2\right) \approx$ 3.66386 . 


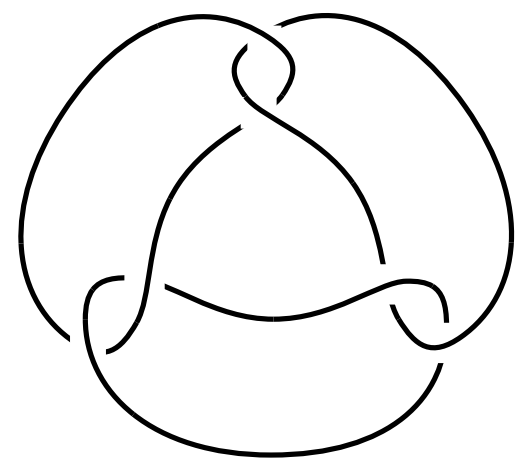

FIGURE 12. Chain-link with three components.

3b. The 5 -braid $\sigma_{1} \sigma_{2}^{2} \sigma_{3} \sigma_{4}$ reaches both $\lambda_{10}\left(D_{5} ; 3\right) \approx$ 2.08102 and $\operatorname{vol}_{10}\left(D_{5} ; 3\right)=\operatorname{vol}\left(S^{3} \backslash C_{3}\right)$.

4a. The 6-braid $\sigma_{1}^{3} \sigma_{2} \sigma_{3} \sigma_{4} \sigma_{5}$ reaches both $\lambda_{11}\left(D_{6} ; 2\right) \approx$ 1.8832 and $\operatorname{vol}_{11}\left(D_{6} ; 2\right) \approx 4.41533$.

4b. The 6-braid $\sigma_{1}^{3} \sigma_{2} \sigma_{1}^{2} \sigma_{3} \sigma_{2} \sigma_{4} \sigma_{5}$ reaches both $\lambda_{11}\left(D_{6} ; 3\right)=\lambda\left(\beta_{5}\right)$ and $\operatorname{vol}_{11}\left(D_{6} ; 3\right)=\operatorname{vol}\left(S^{3} \backslash C_{3}\right)$.

Remark 5.2. There are braids plotted in Figure 11 other than those we identified in the list of observations above. We have experimentally verified by SnapPea that all the 3 -cusped mapping tori in the plots are homeomorphic to either $S^{3} \backslash C_{3}$ or its mirror image, and the other mapping tori having two cusps are results of some Dehn surgeries on $S^{3} \backslash C_{3}$. On the other hand, the mapping tori listed in items $1 \mathrm{~b}, 2 \mathrm{~b}, 3 \mathrm{~b}$, and $4 \mathrm{~b}$ are in fact rigorously shown to be homeomorphic to $S^{3} \backslash C_{3}$ in [Kin and Takasawa 09].

Remark 5.3. The 5 -braids

$$
\beta_{5} \quad \text { and } \quad \beta_{5}^{\prime}=\sigma_{1}^{4} \sigma_{2} \sigma_{3} \sigma_{1} \sigma_{2} \sigma_{3} \sigma_{4}
$$

realize the minimal dilatation $\lambda\left(D_{5}\right)$, but $\operatorname{vol}\left(D_{5}\right)=$ $\operatorname{vol}\left(\beta_{5}\right)<\operatorname{vol}\left(\beta_{5}^{\prime}\right)$. This example says that the mapping class with minimal dilatation does not always realize the minimal volume.

\section{LOWER BOUNDS FOR ENT/VOL}

\subsection{More Problems}

The first half of Theorem 3.2 shows that there exists a constant $B=B(\Sigma)$ such that the inequality $B \leq$ $\operatorname{ent}(\phi) / \operatorname{vol}(\phi)$ holds for any $\phi \in \mathcal{M}^{\mathrm{pA}}(\Sigma)$. However, it is not quite obvious how to find an accurate value of $B$. In

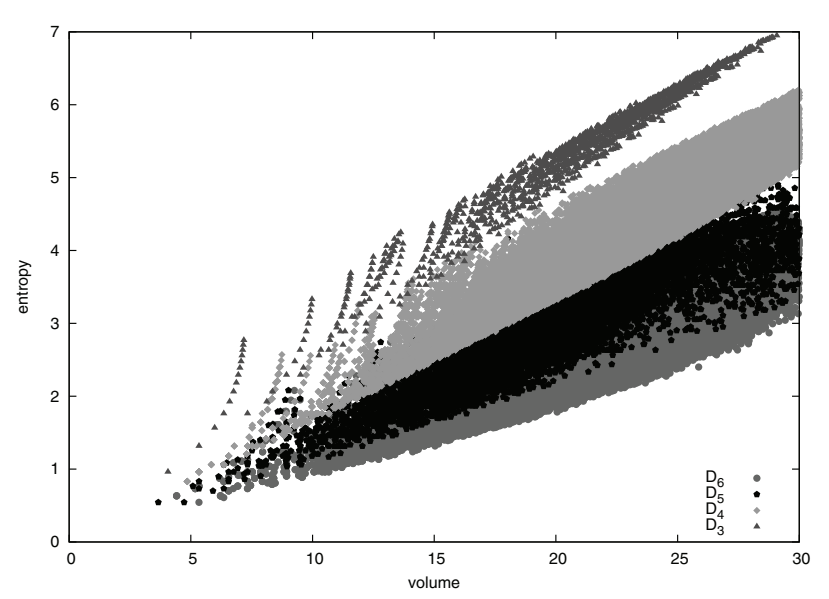

FIGURE 13. Entropy versus volume for $D_{3}, D_{4}, D_{5}$, and $D_{6}$.

this subsection, we formulate a few problems concerning this constant. Let us set

$$
\begin{gathered}
\mathcal{I}(\Sigma)=\inf \left\{\operatorname{ent}(\phi) / \operatorname{vol}(\phi) \mid \phi \in \mathcal{M}^{\mathrm{pA}}(\Sigma)\right\}, \\
\mathcal{I}_{k}(\Sigma)=\min \left\{\operatorname{ent}(\phi) / \operatorname{vol}(\phi) \mid \phi \in \mathcal{M}^{\mathrm{pA}}(\Sigma)\right. \\
\text { of word length } \leq k\},
\end{gathered}
$$

where we fix preferred generating sets as in the previous section.

When the complexity of $\Sigma$ goes higher, the minimal entropy $\operatorname{ent}(\Sigma)$ approaches zero, while the minimal volume $\operatorname{vol}(\Sigma)$ stays bounded away from zero. Thus the bounding constant $B$ in Theorem 3.2 for $D_{n}$ (or $\Sigma_{g, 0}$ ) necessarily tends to zero as $n$ (or $g$ ) goes to $\infty$. We simultaneously plot the data of $\{(\operatorname{ent}(\phi), \operatorname{vol}(\phi))\}$ for $D_{n}$, $3 \leq n \leq 6$, in Figure 13. It is natural to ask for monotonicity.

Problem 6.1. Is it true that $\mathcal{I}\left(D_{n}\right)>\mathcal{I}\left(D_{n+1}\right)$ for all $n \geq 3$ ? Is it true that $\mathcal{I}\left(\Sigma_{g, 0}\right)>\mathcal{I}\left(\Sigma_{g+1,0}\right)$ for all $g \geq 2$ ?

Normalizing the entropy by multiplying area $\left(D_{n}\right)=$ $2 \pi(n-1)$, in Figure 14 we again simultaneously plot the data up to some word lengths. Looking at this plot, one may ask the following question.

Problem 6.2. Does the minimal normalized ratio $2 \pi(n-1) \mathcal{I}\left(D_{n}\right)$ converge to some positive constant as $n$ goes to $\infty$ ?

Also one may ask the following.

Problem 6.3. Does there exist a mapping class $\phi \in$ $\mathcal{M}^{\mathrm{pA}}(\Sigma)$ that attains $\mathcal{I}(\Sigma)$ ? 


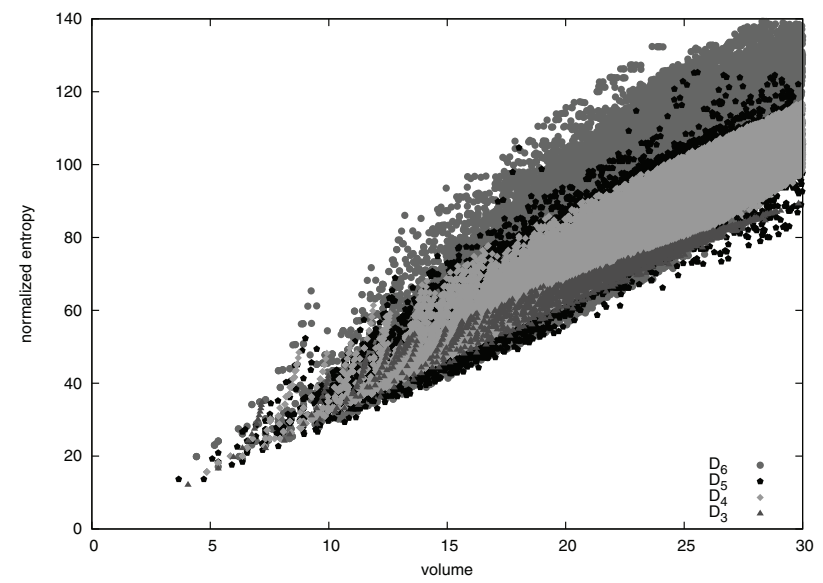

FIGURE 14. Normalized entropy versus volume for $D_{3}, D_{4}, D_{5}$, and $D_{6}$.

To study Problems 6.1, 6.2, and 6.3, we computed $\mathcal{I}_{k}(\Sigma)$ for $\Sigma=D_{n}, 3 \leq n \leq 6$ (Figure 15) and their normalized values (Figure 16). We observe that $\mathcal{I}_{k}\left(D_{3}\right)$ is achieved by the mapping class $\sigma_{1} \sigma_{2}^{-1}$ up to $k=15$. On the other hand, $\mathcal{I}_{k}(\Sigma)$ decreases as $k$ increases for the other surfaces. We thus propose the following conjecture.

\section{Conjecture 6.4.}

1.

$$
\mathcal{I}\left(D_{3}\right)=\frac{\operatorname{ent}\left(\sigma_{1} \sigma_{2}^{-1}\right)}{\operatorname{vol}\left(\sigma_{1} \sigma_{2}^{-1}\right)} \approx 0.2370
$$

2. There are no mapping classes that attain $\mathcal{I}\left(D_{n}\right)$ for $n \geq 4$.

In contrast with Problem 6.1, the graph in Figure 16 suggests that the normalized ratio may not be monotone as $n$ increases.

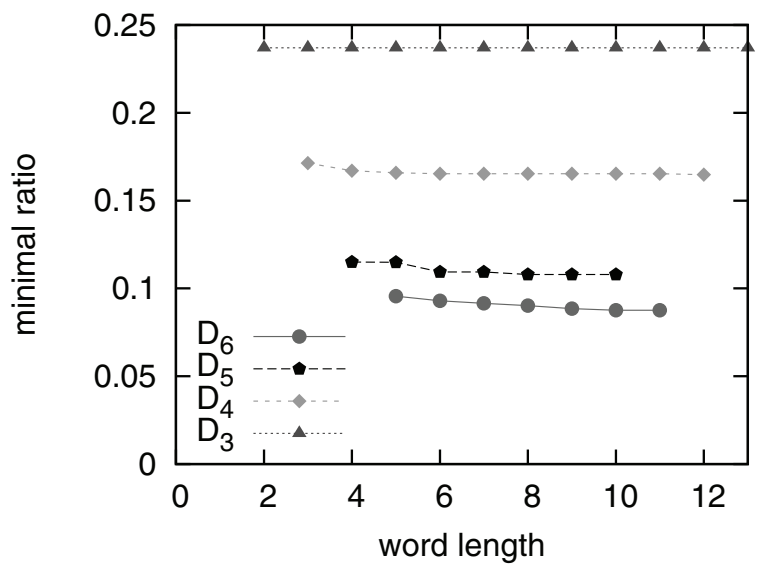

FIGURE 15. Minimal ratio.

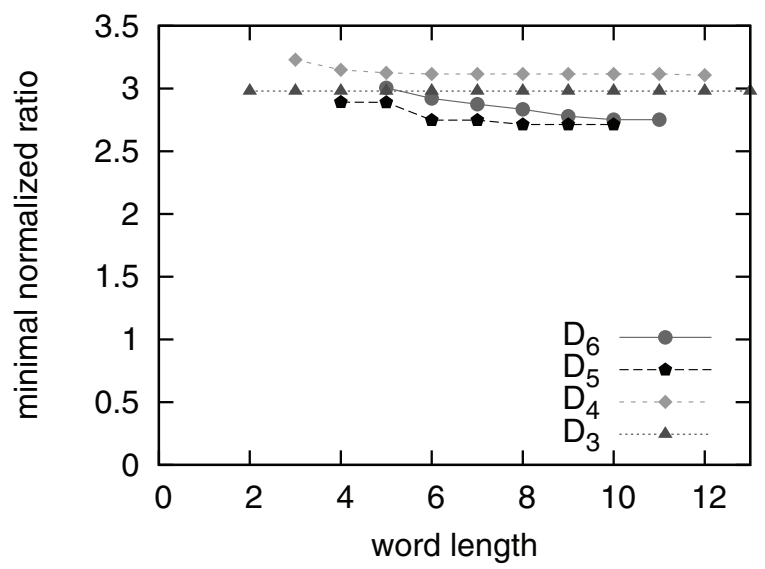

FIGURE 16. Minimal normalized ratio.

Remark 6.5. The related normalized quantity $2(n-1) \pi \operatorname{ent}\left(D_{n}\right)$ is not monotone. In fact, we see from Table 1 that if $3 \leq n \leq 5$, this quantity attains the largest value at $n=4$.

\subsection{A Lower Bound for $\mathcal{I}\left(\Sigma_{1,1}\right)$}

Recall that we chose a preferred generating set $\left\{\tau_{1}, \tau_{2}\right\}$ for $\mathcal{M}\left(\Sigma_{1,1}\right)$ in Section 4. The following result is well known.

Lemma 6.6. Any pseudo-Anosov $\phi \in \mathcal{M}^{\mathrm{pA}}\left(\Sigma_{1,1}\right)$ is conjugate to a mapping class

$$
\tau_{1}^{m_{1}} \tau_{2}^{-n_{1}} \cdots \tau_{1}^{m_{\ell}} \tau_{2}^{-n_{\ell}},
$$

where $\ell, m_{i}$, and $n_{i}$ are some positive integers. A presentation of the mapping class $\tau_{1}^{m_{1}} \tau_{2}^{-n_{1}} \cdots \tau_{1}^{m_{\ell}} \tau_{2}^{-n_{\ell}}$ in this form is unique up to cyclic permutations. Conversely, every mapping class of the form (6-1) is pseudo-Anosov.

The integer $\ell$ in the above form is called the block length of $\phi$.

Theorem 6.7. For each $\phi \in \mathcal{M}^{\mathrm{pA}}\left(\Sigma_{1,1}\right)$, we have

$$
\frac{\operatorname{ent}(\phi)}{\operatorname{vol}(\phi)}>\frac{\log \left(\frac{3+\sqrt{5}}{2}\right)}{2 v_{8}} \approx 0.1313
$$

where $v_{8} \approx 3.6638$ is the volume of a regular ideal octahedron.

Proof: It is well known that the mapping class group $\mathcal{M}\left(\Sigma_{1,1}\right)$ is isomorphic to $\operatorname{SL}(2, \mathbb{Z})$ and the dilatation of a pseudo-Anosov map corresponds to the largest real eigenvalue of the matrix representative. 
We set

$$
M_{1}=\left(\begin{array}{ll}
1 & 1 \\
0 & 1
\end{array}\right) \quad \text { and } \quad M_{2}=\left(\begin{array}{cc}
1 & 0 \\
1 & 1
\end{array}\right) .
$$

These are the matrix representatives for $\tau_{1}$ and $\tau_{2}^{-1}$ respectively. Suppose that $\phi=\tau_{1}^{m_{1}} \tau_{2}^{-n_{1}} \cdots \tau_{1}^{m_{\ell}} \tau_{2}^{-n_{\ell}}$ is a pseudo-Anosov map of block length $\ell$. Then

$$
M=M_{1}^{m_{1}} M_{2}^{n_{1}} M_{1}^{m_{2}} M_{2}^{n_{2}} \cdots M_{1}^{m_{\ell}} M_{2}^{n_{\ell}}
$$

is the matrix representative for $\phi$. Since $M \geq\left(M_{1} M_{2}\right)^{\ell}$, the largest eigenvalue of $M$ is greater than that of $\left(M_{1} M_{2}\right)^{\ell}$. We thus have

$$
\lambda(\phi) \geq \lambda\left(\left(\tau_{1} \tau_{2}^{-1}\right)^{\ell}\right)=\left(\frac{3+\sqrt{5}}{2}\right)^{\ell} .
$$

On the other hand, using [Agol 03, Corollary 2.4], we have

$$
\operatorname{vol}(\phi)<2 \ell v_{8} .
$$

Hence

$$
\frac{\operatorname{ent}(\phi)}{\operatorname{vol}(\phi)}>\frac{\ell \cdot \log \left(\frac{3+\sqrt{5}}{2}\right)}{2 \ell v_{8}}=\frac{\log \left(\frac{3+\sqrt{5}}{2}\right)}{2 v_{8}} \approx 0.1313 .
$$

With the aid of SnapPea, one can have a more accurate estimate for some special cases.

Proposition 6.8. For each $\phi \in \mathcal{M}^{\mathrm{pA}}\left(\Sigma_{1,1}\right)$ of block length 1 , we have

$$
\frac{\operatorname{ent}(\phi)}{\operatorname{vol}(\phi)} \geq \frac{\operatorname{ent}\left(\tau_{1} \tau_{2}^{-1}\right)}{\operatorname{vol}\left(\tau_{1} \tau_{2}^{-1}\right)}=\frac{\log \left(\frac{3+\sqrt{5}}{2}\right)}{2 v_{3}} \approx 0.4741 .
$$

Proof: Let $d$ be the constant on the right-hand side of $(6-3)$. If $\operatorname{ent}(\phi) \geq d \cdot 2 v_{8}$, then $\operatorname{ent}(\phi) / \operatorname{vol}(\phi)>d$, since $\operatorname{vol}(\phi)<2 v_{8}($ see $(6-2))$. Set

$$
\begin{gathered}
Y=\left\{\phi \in \mathcal{M}^{\mathrm{pA}}\left(\Sigma_{1,1}\right) \text { of block length } 1 \mid\right. \\
\left.\operatorname{ent}(\phi)<d \cdot 2 v_{8}<3.4748\right\} .
\end{gathered}
$$

This is a finite set.

When $\phi=\tau_{1}^{m} \tau_{2}^{-n}, \lambda(\phi)$ is the largest eigenvalue of

$$
\left(\begin{array}{cc}
1+m n & m \\
n & 1
\end{array}\right)
$$

that is,

$$
\lambda(\phi)=\frac{2+m n+\sqrt{4 m n+(m n)^{2}}}{2} .
$$

If $\tau_{1}^{m} \tau_{2}^{-n} \in Y$, then $\lambda(\phi)<e^{3.4748}<33$. Hence we have $m n \leq 31$. Computing ent $(\phi) / \operatorname{vol}(\phi)$ for each $\phi=\tau_{1}^{m} \tau_{2}^{-n}$ with $m n \leq 31$ by SnapPea, we see that it is greater than or equal to $d$.
Remark 6.9. The same strategy in the proof of Proposition 6.8 works for cases with a few more longer block lengths. However, the computational cost becomes larger and larger.

We thus make the following conjecture.

\section{Conjecture 6.10.}

$$
\mathcal{I}\left(\Sigma_{1,1}\right)=\frac{\operatorname{ent}\left(\tau_{1} \tau_{2}^{-1}\right)}{\operatorname{vol}\left(\tau_{1} \tau_{2}^{-1}\right)} \approx 0.4741 .
$$

We conclude this paper with the following remark.

Remark 6.11. It is well known that a mapping class $\phi$ of $\mathcal{M}\left(D_{3}\right)$ can be lifted to $\tilde{\phi}$ of $\mathcal{M}\left(\Sigma_{1,1}\right)$ and that the following identities hold:

$$
\operatorname{ent}(\phi)=\operatorname{ent}(\tilde{\phi}) \quad \text { and } \quad \operatorname{vol}(\phi)=2 \operatorname{vol}(\tilde{\phi}) .
$$

See, for instance, [Guéritaud 06]. Hence Conjecture 6.10 is equivalent to the first statement of Conjecture 6.4.

\section{ACKNOWLEDGMENTS}

We would like to thank Kazuhiro Ichihara, Masaharu Ishikawa, and Kenneth J. Shackleton for many discussions and conversations, and Shigenori Matsumoto for helpful comments. We also would like to thank the anonymous referees for valuable suggestions.

The first author is partially supported by Grant-in-Aid for Young Scientists (B) (No. 20740031), MEXT, and the second author is partially supported by Scientific Research (A) (No. 18204004), JSPS, Japan.

\section{REFERENCES}

[Agol 03] I. Agol. "Small 3-Manifolds of Large Genus." Geometriae Dedicata 102 (2003), 53-64.

[Agol 08] I. Agol. "The Minimal Volume Orientable Hyperbolic 2-Cusped 3-Manifolds." arXiv:math.GT/0804.0043, 2008.

[Brock 03] J. Brock. "Weil-Petersson Translation Distance and Volumes of Mapping Tori." Communication in Analysis and Geometry 11 (2003), 987-999.

[Brock et al. 09] J. Brock, H. Mazur, and Y. Minsky. "Asymptotics of Weil-Petersson Geodesics II: Bounded Geometry and Bounded Entropy." In preparation, 2009.

[Cho and Ham 08] J. Cho and J. Ham. "The Minimal Dilatation of a Genus-Two Surface." Experimental Mathematics 17 (2008), 257-269.

[Fathi 87] A. Fathi. "Dehn Twists and Pseudo-Anosov Diffeomorphisms." Inventions Mathematicae 87 (1987), 129-151. 
[Fathi et al. 79] A. Fathi, F. Laudenbach, and V. Poenaru. "Travaux de Thurston sur les surfaces." Asterisque 66-67 (1979), 159-175.

[Gardiner and Lakic 00] F. Gardiner and N. Lakic. Quasiconformal Teichmüller Theory, AMS Mathematical Surveys and Monographs 76. Providence: AMS, 2000.

[Guéritaud 06] F. Guéritaud, with an appendix by D. Futer. "On Canonical Triangulations of Once-Punctured Torus Bundles and Two-Bridge Link Complements." Geometry and Topology 10 (2006), 1239-1284.

[Ham and Song 07] J. Y. Ham and W. T. Song. "The Minimum Dilatation of Pseudo-Anosov 5-Braids." Experimental Mathematics 16 (2007), 167-179.

[Ivanov 90] N. V. Ivanov. "Stretching Factors of PseudoAnosov Homeomorphisms." Zap. Nauchn. Sem. Leningrad. Otdel. Mat. Inst. Steklov (LOMI) 167 (1988) 111-116; translation in Journal of Soviet Mathematics 52 (1990), 2819-2822.

[Kin and Takasawa 09] E. Kin and M. Takasawa. "PseudoAnosov Braids with Small Entropy and the Magic 3Manifold." Preprint,2009.

[Ko et al. 02] K. H. Ko, J. Los, and W. T. Song. "Entropies of Braids." Journal of Knot Theory and Its Ramifications 11 (2002), 647-666.

[Linch 74] M. Linch. "A Comparison of Metrics on Teichmüller Space." Proceedings of the American Mathematical Society 43 (1974), 349-352.

[Long and Morton 86] D. Long and H. Morton. "Hyperbolic 3-Manifolds and Surface Automorphisms." Topology 25 (1986), 575-583.
[Matsuoka 86] T. Matsuoka. "Braids of Periodic Points and 2-Dimensional Analogue of Shorkovskii's Ordering." In Dynamical Systems and Nonlinear Oscillations, edited by G. Ikegami, pp. 58-72. River Edge, NJ: World Scientific, 1986 .

[Minsky 93] Y. Minsky. "Teichmüller Geodesics and Ends of Hyperbolic 3-Manifolds." Topology 32 (1993), 625-647.

[Otal and Kay 01] J.-P. Otal and L. Kay. The Hyperbolization Theorem for Fibered 3-Manifolds, SMF/AMS Texts and Monographs 7. Providence: American Mathematical Society, 2001.

[Royden 74] H. Royden. "Invariant Metric on Teichmüller Space." In Contributions to Analysis: A Collection of Papers Dedicated to Lipman Bers, edited by L. V. Ahlfors. New York: Academic Press, 1974.

[Takasawa 00] M. Takasawa. "Computing Invariants of Mapping Classes of Surfaces." PhD thesis, Tokyo Institute of Technology, 2000.

[Thurston 79] W. Thurston. "The Geometry and Topology of 3-Manifolds." Lecture Notes, Princeton University, 1979.

[Thurston 88] W. Thurston. "On the Geometry and Dynamics of Diffeomorphisms of Surfaces." Bulletin of the American Mathematical Society 19 (1988), 417-431.

[Thurston 98] W. Thurston. "Hyperbolic Structures on 3Manifolds II: Surface Groups and 3-Manifolds Which Fiber over the Circle." Preprint, arXiv:math/9801045v1, 1998.

[Walters 82] P. Walters. An Introduction to Ergodic Theory. New York: Springer-Verlag, 1982.

Eiko Kin, Department of Mathematical and Computing Sciences, Tokyo Institute of Technology, Ohokayama, Meguro, Tokyo 152-8552, Japan (kin@is.titech.ac.jp)

Sadayoshi Kojima, Department of Mathematical and Computing Sciences, Tokyo Institute of Technology, Ohokayama, Meguro, Tokyo 152-8552, Japan (sadayosi@is.titech.ac.jp)

Mitsuhiko Takasawa, Department of Mathematical and Computing Sciences, Tokyo Institute of Technology, Ohokayama, Meguro, Tokyo 152-8552, Japan (takasawa@is.titech.ac.jp)

Received December 23, 2008; accepted in revised form February 17, 2009. 